\title{
EDITORIAL
}

\section{Hematopoietic stem cell transplantation-associated thrombotic microangiopathy: defining a disorder}

\author{
Bone Marrow Transplantation (2008) 41, 917-918; \\ doi:10.1038/bmt.2008.7
}

Hematopoietic stem cell transplantation (HSCT)-associated thrombotic microangiopathy (TMA), abbreviated as TA-TMA, was described by Batts and Lazarus ${ }^{1}$ in a recent issue of Bone Marrow Transplantation as an infrequent but frustrating complication of HSCT. However, other clinicians caring for patients undergoing HSCT, including Marks and Bird in their correspondence in this issue, describe TA-TMA as more common and more critical than implied by Batts and Lazarus. ${ }^{1}$ These comments emphasize the importance of TA-TMA. The importance is documented not only by the experience of clinicians but also by extensive clinical investigation ${ }^{1}$ and efforts to develop consensus diagnostic criteria. ${ }^{2,3}$ Despite these efforts, the syndrome of TA-TMA has resisted clear definition, accurate diagnosis and effective treatment.

A fundamental question has been: 'Does TA-TMA actually exist as a discrete clinical entity?' Does it deserve to be a stand-alone syndrome, or is TA-TMA merely a manifestation of multiple other well-recognized complications of HSCT, such as GVHD and infections? These iconoclastic questions reflected my perspective as a hematologist, whose experience with TA-TMA was from consultations for patients with possible thrombotic thrombocytopenic purpura (TTP) following HSCT, for whom plasma exchange treatment was considered. These patients were always critically ill; treatment with plasma exchange seemed ineffective; most patients died; six patients had autopsies; all had died of disseminated infection (Aspergillus, 3; Candida, 2 and Staphylococcus epidermidis, 1). Our own experience seemed to be consistent with other case series. ${ }^{4}$ Therefore, my opinion 4 years ago was that TATMA was not a distinct clinical entity. ${ }^{4}$ At that time my glib quip to my transplant clinician colleagues was that a diagnosis of TTP following HSCT was the first sign of disseminated aspergillosis.

But my opinion changed. We had considered TA-TMA to be a 'TTP-like syndrome'; ${ }^{5}$ now I think that was unfair and inappropriate, but perhaps it was inevitable. Inevitable because the initial descriptions of TA-TMA in $1980^{6}$ and $1981^{7}$ initiated observations that coincided with the clinical trial performed between 1982 and 1989, which documented the effectiveness of plasma exchange in patients with TTP. ${ }^{8}$ This clinical trial ${ }^{8}$ transformed TTP. The previous pentad of clinical features, developed during the era before effective treatment, ${ }^{9}$ became obsolete; only thrombocytopenia and microangiopathic hemolytic anemia were now required for the diagnosis ${ }^{8}$ and many patients had only these two abnormalities. ${ }^{10}$ The availability of effective treatment for TTP required urgent diagnosis and resulted in decreased stringency of diagnostic criteria; this in turn resulted in a 10-fold increase in the apparent incidence of TTP. ${ }^{11}$ TA-TMA was caught up in this rising tide of diagnoses of TTP and optimism about plasma exchange treatment, because thrombocytopenia and microangiopathic hemolytic anemia were also its constant features. But absence of response to plasma exchange was a disappointing difference from TTP.

My opinion changed because of our recent analysis of autopsies on all 20 patients who died following HSCT at the University of Oklahoma, between 1994 and 2005. ${ }^{12}$ Six of these patients had been clinically diagnosed with TATMA (we had originally called the diagnosis TTP and had treated them with plasma exchange, as described above) based on the presence of thrombocytopenia in spite of engraftment, microangiopathic hemolytic anemia with prominent red cell fragmentation, inappropriately high levels of serum lactate dehydrogenase and often neurologic abnormalities and renal failure without an apparent etiology. When we began this study, I assumed that TATMA was merely a manifestation of multiple other complications of HSCT, ${ }^{4}$ such as acute GVHD or infection with an angioinvasive microorganism, such as Aspergillus, that can mimic all clinical features of TTP. ${ }^{13}$ I predicted that the pathologic abnormalities would not distinguish the 6 patients who were clinically diagnosed as having TATMA from the 14 patients who were not. I was wrong. Our transplant clinicians had been right. They did not have the advantage of the recent, more quantitative diagnostic criteria $^{2,3}$ but their judgments, based on the same clinical features used by these two groups, ${ }^{2,3}$ were proven correct by the pathologic correlation. ${ }^{12}$ All six patients with a clinical diagnosis of TA-TMA had extensive renal TMA; only 2 of the other 14 patients, who had not been clinically diagnosed with TA-TMA, had histologic evidence of TMA; the difference was significant $(P<0.0001)$. TA-TMA was not considered in the two patients with renal TMA, who were not diagnosed with TA-TMA because of the presence of other overt complications, grade 4 GVHD and sepsis. ${ }^{12}$ The autopsy data were distinct from TTP because the TMA was essentially limited to the kidney; three of the six patients with clinical features of TA-TMA also had rare vessels demonstrating TMA in the pancreas, lung and heart. ${ }^{12}$ These data persuaded me that TA-TMA is a distinct clinical entity that could be clinically diagnosed and the diagnosis could be confirmed by histologic evidence. However, even with evidence supporting TA-TMA as a 
distinct clinical entity, patients with TA-TMA will often have multiple other transplant-associated complications, the mortality rate will be high, and death will most commonly be caused by sepsis and acute GVHD. ${ }^{5}$

The separation of TA-TMA from TTP is an important advance. Names matter. The name TTP implies the need for plasma exchange treatment, which is now considered to be ineffective in TA-TMA. The name TA-TMA no longer triggers consideration of plasma exchange treatment, a procedure with a high rate of major complications. ${ }^{14}$ Simply by having its own name, TA-TMA gains greater recognition. Having its name supported by histologic evidence adds further credibility to TA-TMA as a distinct clinical entity. Although the pathologic observation of renal TMA is associated with multiple disorders, such as malignant hypertension and scleroderma in addition to TTP and hemolytic uremic syndrome (HUS), ${ }^{15}$ TA-TMA is now added to the list of syndromes that cause this pattern of microvascular pathology.

The correlation of the clinical diagnosis of TA-TMA with the histologic evidence of TMA is an important step to support the development of diagnostic criteria. But, like TTP, accurate diagnosis of TA-TMA will be difficult. In contrast to TTP, in which the stringency of diagnostic criteria has been decreased to increase the sensitivity of diagnosis, the proposed diagnostic criteria for TA-TMA have gone in the opposite direction of increased diagnostic stringency to provide a more specific diagnosis. ${ }^{2,3}$ Increased stringency is necessary because of the common concurrent complications in patients following HSCT. These two independent efforts ${ }^{2,3}$ are similar and provide quantitative parameters to aid the clinical judgment of transplant clinicians. These criteria now need to be validated by incorporation into multicenter prospective clinical trials to determine if consistent, comparable cohorts of patients can be identified. If patients can be identified, then the clinical course can be determined and the effects of new treatments can be assessed. The ability of the diagnostic criteria to support clinical trials will establish their value and encourage their acceptance by transplant clinicians, just as the new diagnostic criteria for TTP, established by the Canadian Apheresis Group for their landmark clinical trial, ${ }^{8}$ became the standard for diagnosis. ${ }^{16}$

JN George Colleges of Public Health and Medicine, The University of Oklahoma Health Sciences Center, Oklahoma City, OK, USA E-mail: james-george@ouhsc.edu

\section{References}

1 Batts ED, Lazarus HM. Diagnosis and treatment of transplantation-associated thrombotic microangiopathy: real progress or are we still waiting? Bone Marrow Transplant 2007; 40: 709-719.

2 Ho VT, Cutler C, Carter S, Martin P, Adams R, Horowitz M et al. Blood and marrow transplant clinical trials network toxicity committee consensus summary: thrombotic microangiopathy after hematopoietic stem cell transplantation. Biol Blood Marrow Transplant 2005; 11: 571-575.

3 Ruutu T, Barosi G, Benjamin RJ, Clark RE, George JN, Gratwohl A et al. Diagnostic criteria for hematopoietic stem cell transplantation-associated microangiopathy (TAM): results of a consensus process by an International Working Group. Haematologica 2007; 92: 95-100.

4 George JN, Li X, McMinn JR, Terrell DR, Vesely SK, Selby GB. Thrombotic thrombocytopenic purpura-hemolytic uremic syndrome following allogeneic hematopoietic stem cell transplantation: a diagnostic dilemma. Transfusion 2004; 44: 294-304.

5 Roy V, Rizvi MA, Vesely SK, George JN. Thrombotic thrombocytopenic purpura-like syndromes following bone marrow transplantation: an analysis of associated conditions and clinical outcomes. Bone Marrow Transplant 2001; 27: 641-646.

6 Powles RL, Clink HM, Spence D, Morgenstern G, Watson JG, Selby PJ et al. Cyclosporin A to prevent graft-versus host disease in man after allogeneic bone-marrow transplantation. Lancet 1980; 1: 327-329.

7 Shulman H, Striker G, Deeg HJ, Kennedy M, Storb R, Thomas ED. Nephrotoxicity of cyclosporin A after allogeneic marrow transplantation. $N$ Eng J Med 1981; 305: 1392-1395.

8 Rock GA, Shumak KH, Buskard NA, Blanchette VS, Kelton $\mathrm{JG}$, Nair RC et al. Comparison of plasma exchange with plasma infusion in the treatment of thrombotic thrombocytopenic purpura. $N$ Eng J Med 1991; 325: 393-397.

9 Amorosi EL, Ultmann JE. Thrombotic thrombocytopenic purpura: report of 16 cases and review of the literature. Medicine 1966; 45: 139-159.

10 Vesely SK, George JN, Lämmle B, Studt JD, Alberio L, El-Harake MA et al. ADAMTS13 activity in thrombotic thrombocytopenic purpura-hemolytic uremic syndrome: relation to presenting features and clinical outcomes in a prospective cohort of 142 patients. Blood 2003; 101: 60-68.

11 Clark WF, Garg AX, Blake PG, Rock GA, Heidenhaim AP, Sackett DL. Effect of awareness of a randomized controlled trial on use of experimental therapy. JAMA 2003; 290: 1351-1355.

12 Siami K, Kojouri K, Swisher KK, Selby GB, George JN, Laszik ZG. Thrombotic microangiopathy after allogeneic hematopoietic stem cell transplantation. An autopsy study. Transplantation 2008; 85: 22-28.

13 Robboy SJ, Salisbury K, Ragsdale B, Bobroff LM, Jacobson BM, Colman RW et al. Mechanism of Aspergillus-induced microangiopathic hemolytic anemia. Arch Intern Med 1971; 128: 790-793.

14 Howard MA, Williams LA, Terrell DR, Duvall D, Vesely SK, George JN et al. Complications of plasma exchange in patients treated for clinically suspected thrombotic thrombocytopenic purpura-hemolytic uremic syndrome. III. An additional study of 57 consecutive patients, 2002-2005. Transfusion 2006; 46: $154-156$.

15 Laszik ZG, Silva FG. Hemolytic uremic syndrome, thrombotic thrombocytopenia purpura, and other thrombotic microangiopathies and coagulopathies. In: Jennett JC, Olson JL, Schwartz MM, Silva FG (eds). Heptinstall's Pathology of the Kidney. Lippincott Williams \& Wilkins, Philadelphia, 2007, pp 699-762.

16 George JN. Thrombotic thrombocytopenic purpura. $N$ Eng $J$ Med 2006; 354: 1927-1935. 